\title{
The FOXMI-PLKI axis is commonly upregulated in oesophageal adenocarcinoma
}

\author{
M Dibb ${ }^{1,2}$, N Han', J Choudhury ${ }^{3}$, S Hayes ${ }^{2,3}$, H Valentine ${ }^{4}$, C West ${ }^{4}$, YS Ang ${ }^{2}$ and AD Sharrocks*,I \\ 'Faculty of Life Sciences, University of Manchester, Michael Smith Building, Oxford Road, Manchester MI 3 9PT, UK; ${ }^{2}$ Faculty of Medical and Human \\ Sciences, University of Manchester, Oxford Road, Manchester, UK; ${ }^{3}$ Department of Histopathology, Salford Royal Foundation Trust, Stott Lane, Salford \\ M6 8HD, UK; ${ }^{4}$ School of Cancer and Enabling Sciences, Manchester Academic Health Science Centre, The University of Manchester, Christie Hospital, \\ Manchester, UK
}

BACKGROUND: The transcription factor FOXMI is an important regulator of the cell cycle through controlling periodic gene expression during the G2 and M phases. One key target for FOXMI is the gene encoding the protein kinase PLKI and PLKI itself acts in a positive feedback loop to phosphorylate and activate FOXMI. Both FOXMI and PLKI have been shown to be overexpressed in a variety of different tumour types.

METHODS: We have used a combination of RT-PCR, western blotting, tissue microarrays and metadata analysis of microarray data to study whether the FOXMI-PLKI regulatory axis is upregulated and operational in oesophageal adenocarcinoma.

RESULTS: FOXMI and PLKI are expressed in oesophageal adenocarcinoma-derived cell lines and demonstrate cross-regulatory interactions. Importantly, we also demonstrate the concomitant overexpression of FOXMI and PLKI in a large proportion of oesophageal adenocarcinoma samples. This co-association was extended to the additional FOXMI target genes CCNBI, AURKB and CKSI. In a cohort of patients who subsequently underwent surgery, the expression of several FOXMI target genes was prognostic for overall survival.

CONCLUSIONS: FOXMI and its target gene PLKI are commonly overexpressed in oesophageal adenocarcinomas and this association can be extended to other FOXMI target genes, providing potentially important biomarkers for predicting post-surgery disease survival.

British Journal of Cancer (2012) I 07, 1766-1775. doi:I0.1038/bjc.2012.424 www.bjcancer.com

Published online 4 October 2012

(c) 2012 Cancer Research UK

Keywords: FOXMI; G2-M cell-cycle phase; oesophageal adenocarcinoma; PLKI

Oesophageal cancer is the sixth most common cause of cancer death in the United Kingdom and has a poor prognosis with 5-year survival rates of $<10 \%$ (Coupland et al, 2012). The majority of oesophageal cancers are of the adenocarcinoma histological subtype, the incidence of which was rapidly increasing but has now stabilised (Coupland et al, 2012). Aetiological factors associated with the development of oesophageal adenocarcinoma include the presence of Barrett's metaplasia, obesity, widespread eradication of Helicobacter pylori infection and an ageing population (Koike et al, 2001; Solaymani-Dodaran et al, 2004; Hampel et al, 2005; Adams et al, 2007). The majority of patients present with late-stage disease that is not amenable to endoscopic or surgical resection and have a poor response to conventional systemic chemotherapy (Adams et $a l, 2007)$. Due to the late presentation of the disease, new screening methods that detect oesophageal malignancy at an earlier clinical stage and allow curative therapy are urgently required (Lao-Sirieix et al, 2009). Recent attempts at identifying new prognostic markers for oesophageal adenocarcinomas have focussed on using microarray analysis of mRNA expression

*Correspondence: Dr AD Sharrocks;

E-mail: a.d.sharrocks@manchester.ac.uk

Received 15 June 2012; revised 29 August 2012; accepted 29 August 2012; published online 4 October 2012 patterns, and have led to the identification of four (Peters et al, 2010) and two (Kim et al, 2010) gene signatures which are of prognostic value. Thus, further analysis of gene expression signatures, or the coordinated upregulation of components of molecular pathways might provide further advances in this area.

FOXM1 is a member of the forkhead transcription factor family, which has been shown to have an important role in controlling the cell cycle (Laoukili et al, 2007; Koo et al, 2012). In particular, FOXM1 controls mitotic entry through the periodic upregulation of a group of genes that are maximally expressed as cells progress through late G2 and into M phase (Laoukili et al, 2005). Two of its target genes are CCNB1 and PLK1, and these form part of a kinasedriven positive feedback loop that leads to the phosphorylation of FOXM1 and potentiation of its activity (Major et al, 2004; Fu et al, 2008). Thus, there is an intricate inter-regulatory relationship between FOXM1 and PLK1 that creates a cell-cycle control switch. The link between FOXM1 and cell-cycle control suggests that it is likely to contribute to the aberrant cell proliferation associated with malignancy. Indeed, FOXM1 has been shown to be upregulated in a range of different tumour types (Koo et al, 2012). More recently, FOXM1 was shown to be overexpressed and of potential prognostic significance in oesophageal squamous cell carcinomas (Hui et al, 2012) but before this study, its status in oesophageal adenocarcinomas was unknown. Similarly, the FOXM1 target gene PLK1 has also been shown to be overexpressed 
in a wide range of tumours (Strebhardt and Ullrich, 2006), including oesophageal squamous carcinomas (Feng et al, 2009; Zhao et al, 2010). Molecularly, novel functions for FOXM1 have been identified in cancer cells beyond simply the acceleration of G2-M phase progression (Raychaudhuri and Park, 2011; Koo et al, 2012). This is exemplified by its ability to promote the nuclear translocation of $\beta$-catenin in gliomas, and hence activate a programme of Wnt target genes (Zhang et al, 2011). Together, these observations indicate that FOXM1 and PLK1 are likely central regulators in carcinogenesis and are potential therapeutic targets.

In this study, we investigated the expression of FOXM1 and PLK1 in oesophageal adenocarcinomas with particular emphasis on examining whether there was evidence for co-expression, and hence upregulation of the FOXM1-PLK1 regulatory axis. We show that there is widespread coordinate overexpression of FOXM1 and PLK1 in oesophageal adenocarcinomas, thereby providing the potential for feedback potentiation of FOXM1 activity. Indeed, we extend these studies to demonstrate the coordinate upregulation of a group of additional FOXM1 target genes across a high proportion of oesophageal adenocarcinomas. Some of these target genes appear to be useful prognostic indicators of survival following surgery. PLK1 inhibitors are currently being developed for cancer therapy (Strebhardt and Ullrich, 2006; Lens et al, 2010) and it is likely that cells demonstrating an upregulated FOXM1-PLK1 axis will be particularly susceptible to such treatment.

\section{MATERIALS AND METHODS}

\section{Tissue collection}

Ethical approval was granted by Wrightington Wigan and Leigh Ethics Committee, UK in 2004. Tissue was collected from 70 patients with oesophageal adenocarcinomas, 28 with Barrett's metaplasia and 55 healthy controls. Adenocarcinomas at the gastro-oesophageal junction were classified as oesophageal adenocarcinomas. Age and date at diagnosis, gender, comorbidity, smoking status and survival were recorded. Details of the histological grade of tumour and stage, using the TNM and AJCC criteria were collected. Information on treatments including surgery, chemotherapy, radiotherapy and palliation were also recorded. Biopsy samples, $\sim 4 \mathrm{~mm}$ in size, were taken at the time of endoscopic examination. Biopsy and surgical samples were rapidly frozen in liquid nitrogen and stored at $-80^{\circ} \mathrm{C}$ until needed. Paraffin blocks were used to construct tissue microarrays (TMA) for immunohistochemistry (IHC). Haematoxylin and eosin stained sections from the TMA's were characterised by two expert histopathologists and in the case of adenocarcinoma samples, were determined to contain a large proportion of tumour cells. Frozen biopsy and surgical samples were used for RNA extraction.

\section{Cell lines, cell culture and western analysis}

OE33, Flo1 (oesophageal adenocarcinomas), OE21 (oesophageal squamous cell carcinoma) and Het1A (normal oesophageal) cell lines were grown as described previously (Keld et al, 2010). For nocadazole block experiments, cells were grown for $24 \mathrm{~h}$ and then arrested at G2/M with $100 \mathrm{ng} \mathrm{ml}^{-1}$ nocadazole for $16 \mathrm{~h}$ followed by mRNA or protein extraction. Double thymidine block was performed as described previously (Whitfield et al, 2000). For experiments requiring PLK1 inhibition, $100 \mathrm{~nm}$ R-BI2536 (Activate Scientific GmbH, Prien, Germany) was added $7 \mathrm{~h}$ before harvesting. Cell lysis and western analysis were carried out essentially as described previously (Keld et al, 2010). Following transfer onto a nitrocellulose membrane, proteins were detected with ERK2 (New England Biolabs, Ipswich, ME, USA), FOXM1 (SC-502; Santa Cruz Biotechnology, Santa Cruz, CA, USA) or PLK1 (F-8; Santa Cruz Biotechnology) antibodies.

\section{RNA isolation and RT-PCR analysis}

RNA was extracted, and analysed as described previously (Keld et al, 2010). Real-time RT-PCR was carried out using the primer pairs; FOXM1 (total), ADS2552 (5'-CCTCAAACCCAAACCAGC TA- $\left.3^{\prime}\right)$ and ADS2553 (5'-GAAGCCACTGGATGTTGGAT- $3^{\prime}$ ); PLK1, ADS2550 (5'-AAGAGGAGGAAAGCCCTGAC-3') and ADS2551 (5'-TTCTTCCTCTCCCCGTCATA-3'); FOXM1a, ADS2558 (5'-CCA TAGCAAGCGAGTCCGCA-3'), and ADS2559 (5'-CAGCTAGCAGC ACTGATAAAC- $3^{\prime}$ ); FOXM1b, ADS2562 (5'-CAGCACTGAGAGGA AGCGCA- $\left.3^{\prime}\right)$ and ADS $2563\left(5^{\prime}\right.$-TCGTTTCTGCTGCTTAAAC- $\left.3^{\prime}\right)$; FOXM1c, ADS2560 (5'-CGAGCACTTGGAATCACAGCAGA-3') and ADS2561 (5'-TCCTCAGCTAGCAGCACCTTGG-3'); CCBN1, ADS1728 (5'-GGCCAAAATGCCTATGAAGA- $\left.3^{\prime}\right)$ and ADS1729 (5'-AGATGTTTCCATTGGGCTTG-3'); AURKB, ADS2669 (5'-TGG GACACCCGACATCTTAACGC- $\left.3^{\prime}\right)$ and ADS2670 ( $5^{\prime}$-ACCTTGAGC GCCACGATGAAATGG-3'); 18S, ADS4005 (5'-CGGCTACCACATC CAAGGAA- $\left.3^{\prime}\right)$ and ADS 4006 (5'-GCTGGAATTACCGCGGCT- $\left.3^{\prime}\right)$; GAPDH, ADS2184 (5'-ACAGTCAGCCGCATCTTCTT- $\left.3^{\prime}\right)$ and ADS2185 (5'-TTGATTTTGGAGGGATCTCG-3'). Real-time PCRs were run on a Rotor Gene RG-3000 (Qiagen Ltd, Crawley, UK) and analysed with Rotor-Gene 6 software (Qiagen Ltd). Data are presented relative to $18 \mathrm{~S}$ RNA levels in the same samples. For relative comparison of mRNA levels from tissue specimens, data were further normalised to the level of each gene in a standard concentration of RNA isolated from Hetla cells.

\section{Immunohistochemistry}

Tissue microarrays blocks were constructed as described previously (Keld et al, 2010). Arrays were stained with FOXM1 (SC-500; Santa Cruz Biotechnology) at a $1: 40$ dilution. A negative control slide was created by combining antibody with five-fold excess of blocking peptide (SC-500P) in Tris-buffered saline (TBS) and incubated overnight before adding to sections. Multiple cores for each specimen were constructed and initially scored by MD blinded to the clinical details. Cells were scored on the basis of intensity (score of $0-3$ ) and the percentage of cells stained. The highest score in the triplet of cores was recorded. These two scores were then multiplied to make an overall IHC score. Two histopathologists (JC and SJH), both with a gastrointestinal interest, reviewed a selection of IHC sections, including comparison with positive and negative control sections, in order to confirm accuracy. Tissue microarrays spots which have both normal and tumour cells present only had the tumour-specific part scored. Negative expression was considered to be an IHC score of $<45$, low expression between 46 and 90 , moderate expression 91-150 and high expression was between 151 and 300 .

\section{siRNA and plasmid transfection}

Short interfering (si) RNAs directed against human FOXM1, PLK1 and GAPDH (SMARTpools; Dharmacon, Chicago, IL, USA), and a non-targeting scrambled sequence (Santa Cruz Biotechnology) were used. Cells were transfected as described previously (Keld et al, 2010). siRNA treatment was performed for $48 \mathrm{~h}$ before protein or mRNA expression analysis in an effort to minimise the potentially toxic effects caused by the loss of PLK1.

\section{Microarray analysis}

The recently published microarray data sets profiling gene expression of 28 normal and 64 oesophageal adenocarcinoma samples were downloaded from Gene Expression Omnibus (accession number: GSE13898) (Kim et al, 2010). Pearson's correlation coefficients (PCCs) were computed to identify a set of genes that have similar expression pattern with FOXM1, and 
stringent threshold was applied on the PCC results $(0.8<r$-value) Hierarchal clustering and visualisation of the expression levels of the identified genes were performed by MultiExperiment Viewer, a part of TM4 microarray software suite (Saeed et al, 2006)

\section{RESULTS}

\section{The expression of FOXM1 and PLK1 in oesophageal- derived cell lines}

FOXM1 is generally present in many cell types but its expression is thought to be limited to proliferating cells (Laoukili et al, 2007; Koo et al, 2012). Similarly, its target gene PLK1 is also expressed under these conditions across many cell types (reviewed in Lens et al, 2010). To establish whether FOXM1 and PLK1 are expressed in oesophageal cells, we first determined whether protein could be detected in a range of oesophageal-derived cell lines, including nontumourigenic Hetla cells, and cells originating from squamous carcinomas (OE21) or adenocarcinomas (OE33 and Flo1). For each cell line, asynchronously growing cells, or cells treated with nocodazole to enrich for the G2-M phase population, were analysed by western blotting. All of the cell lines express FOXM1 to similar levels (Figure 1A, upper panel). FOXM1 levels were unaffected by nocodazole treatment, but with the exception of OE33 cells, a lower mobility band was induced by this treatment which is indicative of the expected phosphorylation of FOXM1 that occurs in the G2-M phase (Figure 1A, upper panel, lanes 4-6) (Major et al, 2004). PLK1 was also detected at similar levels across all cell lines, and as expected, its expression was increased upon nocodazole treatment, with the exception of OE33 cells (Figure 1A, lower panel). However, OE33 cells responded poorly to nocodazole treatment, and do not readily accumulate in G2-M (data not shown).

Next, we focussed on oesophageal adenocarcinoma cells and analysed the expression of FOXM1 and PLK1 at the mRNA level. Cells were arrested in early $S$ phase by using a double thymidine block, and then released into fresh media to allow synchronous progression through the cell cycle. FOXM1 expression showed a modest oscillatory pattern during the cell cycle, peaking just before cells accumulated maximally in the G2-M phase of the cell cycle (Figure 1B, top panels). PLK1 expression showed a similar oscillatory pattern, although higher amplitude changes are observed. The kinetics of induction vary between the two cell lines but this reflects the more rapid transition of OE33 cells into the G2-M phase of the cell cycle (Figure 1B, bottom panels).

Together, these results demonstrate that FOXM1 and PLK1 are expressed at both the mRNA and protein levels in oesophagealderived cells, including oesophageal adenocarcinoma cell lines. Generally, there is little difference in their expression among these proliferating cells, although differences in cell-cycle progression are detected among oesophageal adenocarcinoma cell lines.

\section{The FOXM1-PLK1 regulatory circuit is operative in oesophageal adenocarcinoma cells}

FOXM1 controls PLK1 expression and PLK1 forms part of a positive feedback loop to phosphorylate and hyperactivate FOXM1 (Figure 2A; Laoukili et al, 2005; Fu et al, 2008). We therefore examined whether this regulatory circuit is operational in oesophageal adenocarcinoma cells. First, we depleted FOXM1 or PLK1 using siRNA, and examined the expression of each protein. OE33 cells were treated with siRNA constructs for $48 \mathrm{~h}$, at which point, although cell-cycle arrest is apparent, extensive cell death was not observed (data not shown). As expected, FOXM1 siRNA resulted in efficient FOXM1 depletion and PLK1 siRNA caused a loss of PLK1 (Figure 2B). However, FOXKM1 depletion also led to reductions in PLK1 levels, whereas PLK1 depletion also reduced FOXM1 protein expression to some degree. These observations are consistent with the known regulatory properties of FOXM1 as an activator of PLK1 transcription. To confirm that FOXM1 was regulating PLK1 expression at the transcriptional level, we examined PLK1 expression by real-time RT-PCR. Depletion of FOXM1 led to a significant decrease in PLK1 levels (Figure 2C,
A
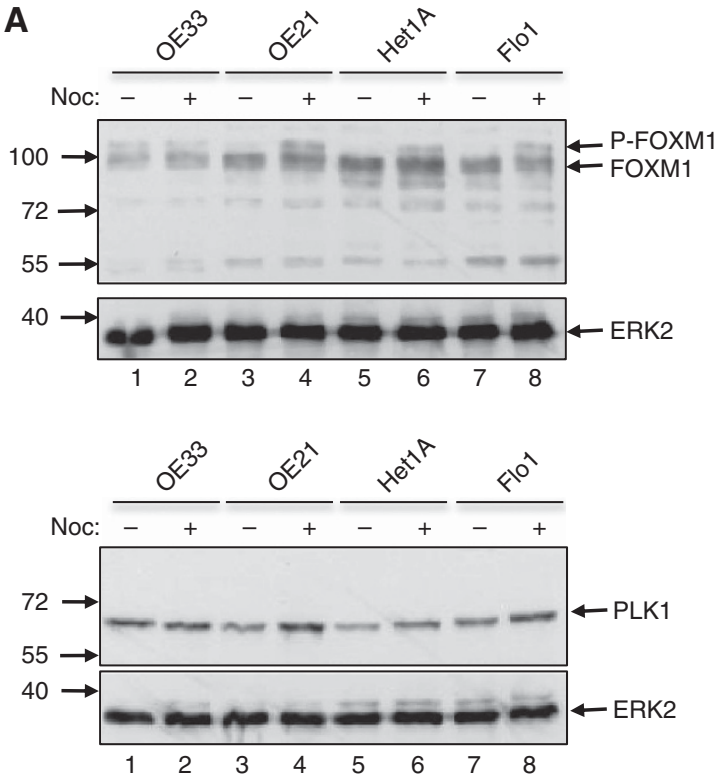

B
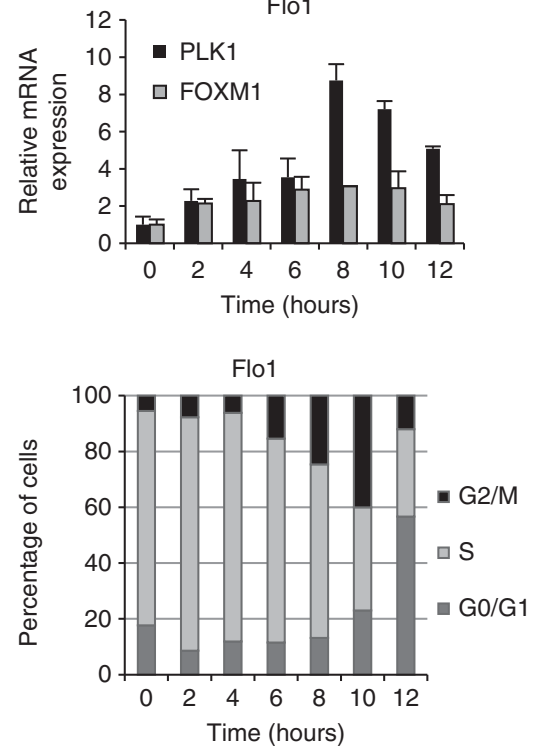
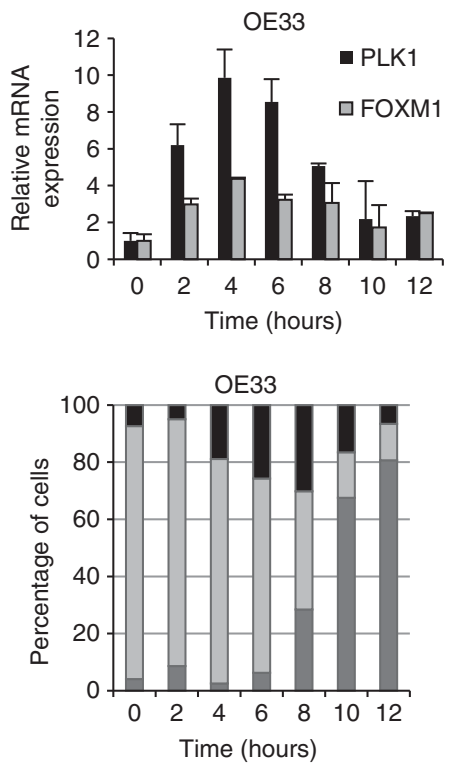

Figure I The expression of FOXMI and PLKI in oesophageal-derived cell lines. (A) Western blot analysis of FOXMI (top panel) and PLKI (bottom panel) protein expression in OE33, OE2 I, Het la and Flol oesophageal cell lines treated with DMSO or with nocadazole (noc) for I6 h. The locations of the molecular weight markers are shown on the left ( $\mathrm{kDa}$ ). The positions of the bands corresponding to FOXMI and PLKI are shown and lower mobility bands consistent with phosphorylation of FOXMI (P-FOXMI) are indicated. ERK2 was used as a loading control. (B) Real-time RT-PCR analysis of FOXMI (grey bars) and PLKI (black bars) expression in Flol (left) and OE33 (right) cells following release for the indicated time points from a double thymidine block (top graphs). The mean expression from two independent experiments relative to time zero (taken as I) is shown. Error bars indicate the standard deviation. The corresponding cell cycle profiles are shown beneath each graph. 
bottom panel). A reciprocal effect of PLK1 depletion was seen on FOXM1 levels (Figure 2C, top panel). Due to the potential indirect effects of PLK1 depletion on cell-cycle properties, we also used a PLK1 inhibitor, BI2536, to cause immediate PLK1 inhibition in cells released from a double thymidine-induced cell-cycle arrest. Long-term treatment with the PLK1 inhibitor leads to the death of many cell types (reviewed in Strebhardt and Ullrich, 2006), including oesophageal adenocarcinoma cells (data not shown), therefore, we limited our gene expression analysis to $7 \mathrm{~h}$ after release from the arrest, as cells progress from late $\mathrm{G} 2$ into $M$ phase. We used this approach to analyse the expression of PLK1 and two other FOXM1 target genes $C C N B 1$ and $A U R K B$, at this point of the cell cycle. All three genes showed reduced expression levels, although the changes in $A U R K B$ expression did not reach statistical significance (Figure 2D). There was also a reduction in FOXM1 mRNA levels. Thus, active PLK1 is required for maximal activation of FOXM1 target genes.

Together, these results indicate that the FOXM1-PLK1 regulatory circuit is intact in oesophageal adenocarcinoma cells, with FOXM1 controlling the expression of PLK1 and other cellcycle target genes, and PLK1 feeding back to accentuate this control.

\section{FOXM1 and PLK1 are overexpressed in oesophageal adenocarcinomas}

Having established the expression of FOXM1 and PLK1 in oesophageal adenocarcinoma-derived cell lines, we next wanted to determine whether their expression differed between normal oesophageal tissue, and samples derived from patients with oesophageal adenocarcinomas. We collected biopsy samples from 19 patients with normal oesophageal tissue, and 69 oesophageal adenocarcinoma samples with 34 matched samples from adjacent normal tissue. An additional 12 samples were obtained from tissues diagnosed histologically to demonstrate features of Barrett's oesophageal tissues and 9 matched samples from adjacent normal tissue. The samples from normal oesophageal tissue provide control reference points for examining the changing expression levels of FOXM1 and PLK1 expression in the adenocarcinoma samples.

First, we analysed the expression of FOXM1 and PLK1 by realtime RT-PCR. Both genes are expressed at low levels in normal oesophageal tissue, but they are both overexpressed in a large proportion of the oesophageal adenocarcinoma samples (Figure $3 \mathrm{~A})$. The average expression levels were three-fold and four-fold over those found in normal tissue for FOXM1 and PLK1, respectively ( $P$-values $<0.001$ ), and $40 \%$ and $53 \%$ of samples express significantly increased levels of FOXM1 and PLK1, respectively. The median level and the overall distribution of expression levels for both genes are significantly higher in the adenocarcinoma samples than in normal tissue or in tissues with Barrett's metaplasia (Figure 3B). There are three different FOXM1 isoforms generated by alternative splicing (Ye et al, 1997). We therefore designed primer pairs to specifically detect different FOXM1 splice forms and analysed whether any particular splice form was overexpressed among the adenocarcinoma samples. In general, elevated levels of FOXM1a and FOXM1b were seen in all cancer samples, whereas there was more heterogeneity with FOXM1c (Figure 3C). However, as expected, very few of the splice forms were overexpressed in samples from normal tissue. Thus,
A

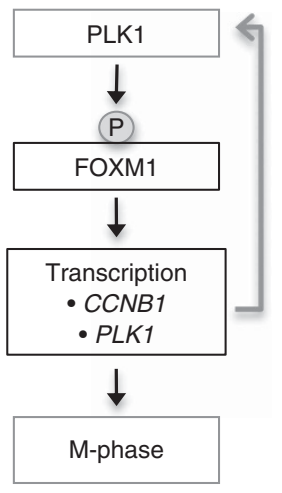

B

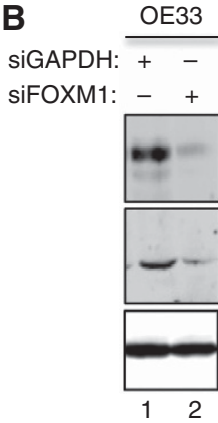

SIGAPDH:

SiPLK1:
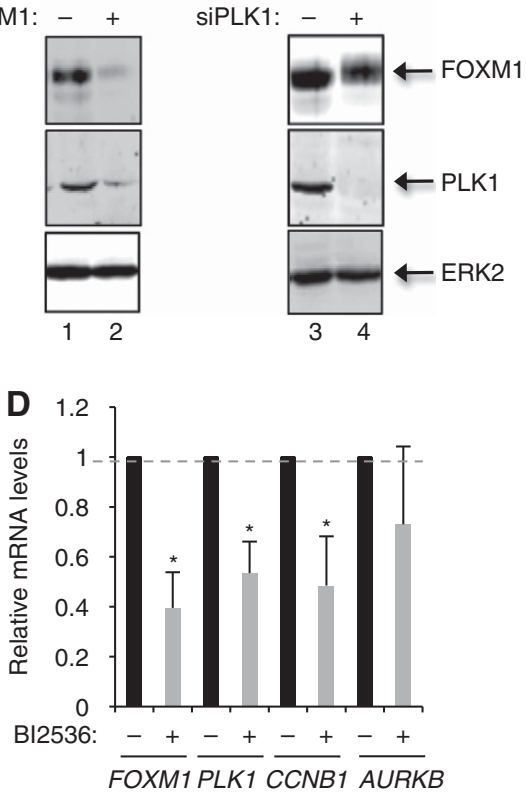

OE33

$+\quad-$

.

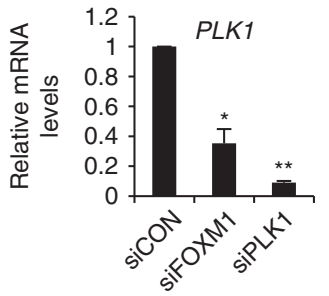

Figure 2 Regulatory inter-relationships between FOXMI and PLKI in oesophageal adenocarcinoma cells. (A) Model of the regulatory inter-relationships between FOXMI and PLKI. FOXMI activates PLKI expression that feeds back to phosphorylate (P) to further enhance FOXMI activity. (B) Western analysis of FOXMI and PLKI protein expression following siRNA-mediated knockdown of FOXMI (left) and PLKI (right) in OE33 cells. Control cells were treated with siRNAs targeting GAPDH. ERK2 is a loading control. The locations of the bands corresponding to each of the proteins are shown on the right. (C) RT-PCR analysis of FOXMI (top) and PLKI (bottom) mRNA expression following siRNA-mediated knockdown of FOXMI and PLKI in OE33 cells. As a control, cells were treated with non-targeting siRNAs (siCON). Data are the average of two independent experiments and presented as the mean and standard deviations of gene expression relative to cells treated with the non-targeting control (taken as 'I'). (D) RT-PCR analysis of the expression of FOXMI mRNA, and its target genes PLKI, CCNBI and AURKB following cell synchronisation with double thymidine treatment and subsequent release into media containing BI2536 or DMSO (negative control). Cells were harvested $7 \mathrm{~h}$ after release. The experiments were repeated three times and data are presented as the mean and standard deviation of the three experiments relative to DMSO-treated cells (taken as I). Statistical significance is indicated on the graphs (*P-value $<0.05$; ** $P$-value $<0.01$ ). 
although there is a clear pattern of FOXM1 overexpression in oesophageal adenocarcinomas, there is no particular preference for a particular splice form.
Having established that FOXM1 and PLK1 are overexpressed in oesophageal adenocarcinomas at the mRNA level, we next analysed their expression at the protein level. First, we used TMAs
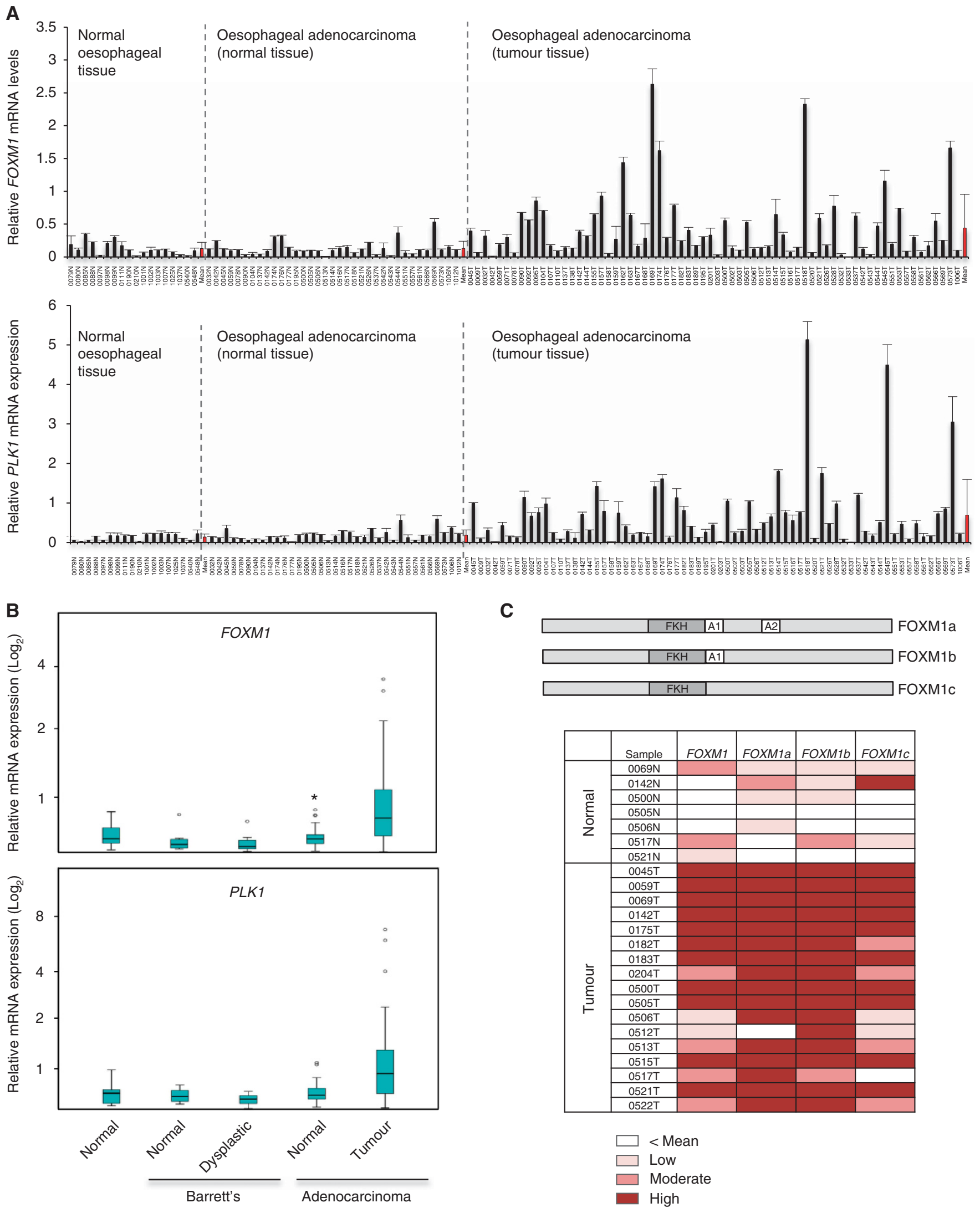

C
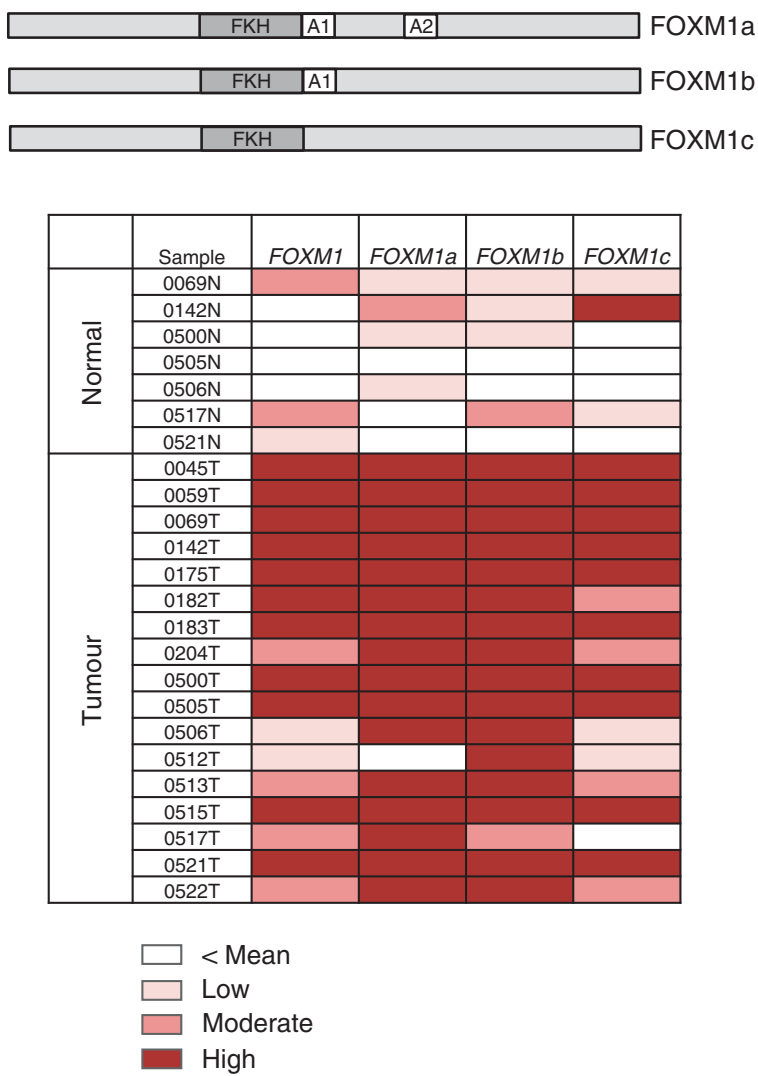
to probe for expression of FOXM1 across a collection of 15 samples from normal tissue and 117 samples from oesophageal adenocarcinomas. A range of different levels of FOXM1 expression were seen among tumour samples (Figure 4A). A significantly higher proportion of oesophageal adenocarcinoma samples expressed moderate or high levels of FOXM1 compared with normal tissue (Figure 4B; $P$-value 0.004), and this was reflected in the higher median and overall distribution of expression scores across the TMA in oesophageal adenocarcinoma samples (Figure 4C). We also attempted to analyse PLK1 expression using TMAs but were unable to find a suitable antibody (data not shown). As an alternative method for determining protein levels, we used western analysis to compare FOXM1 expression across normal and tumour samples. Among seven matched samples, four showed elevated FOXM1 expression in the cancer-derived tissue (Figure 4D, upper panel, lanes 4 and 8; lower panel, lanes 5 and 9); high levels of FOXM1 were also observed in one of two additional tumour samples analysed (Figure 4D, upper panel, lanes 1 and 2). These results are broadly consistent with what is seen in the TMAs and RT-PCR analyses, where $40-60 \%$ of tumour samples express high
A

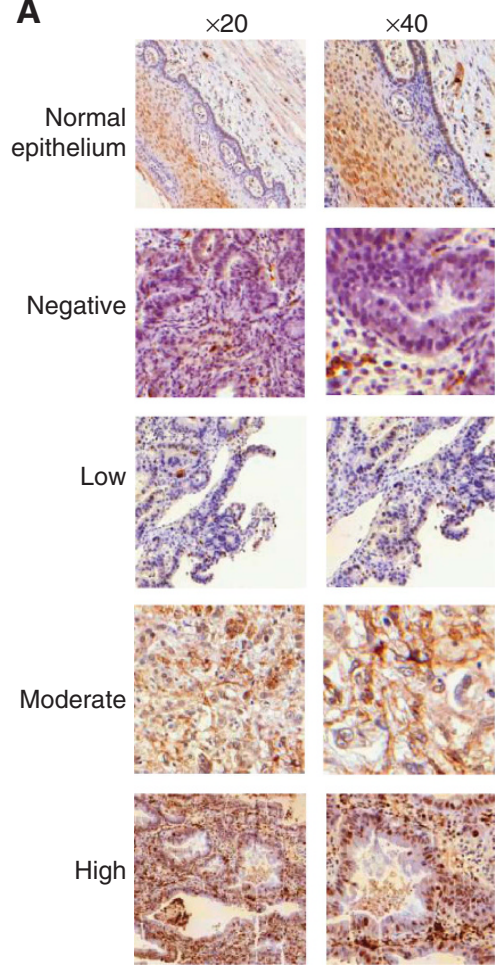

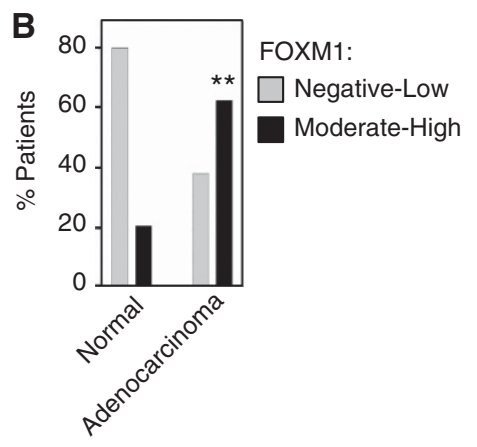

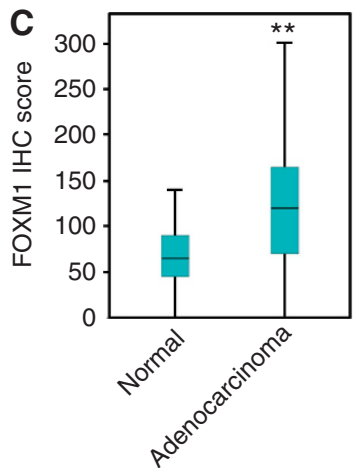

D
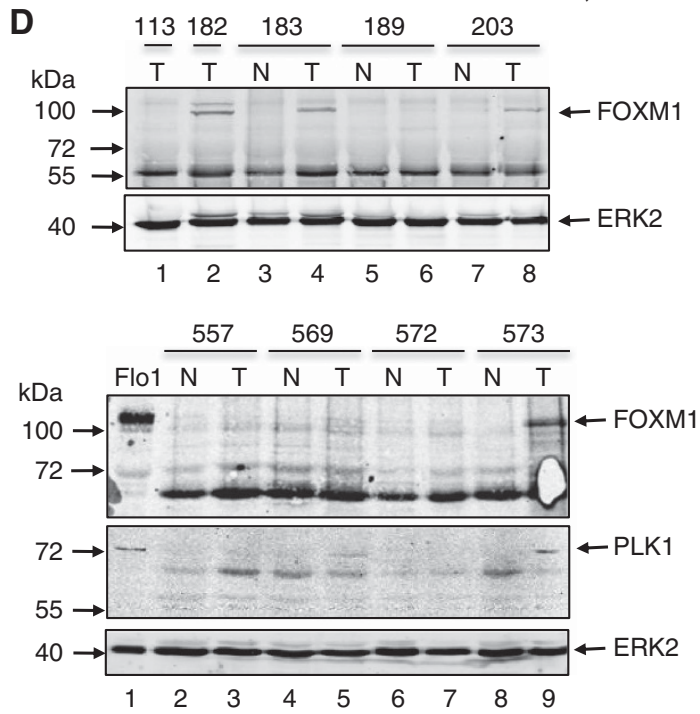

Figure 4 FOXMI and PLKI protein expression in oesophageal adenocarcinomas. (A) Examples of TMA staining for FOXMI protein (brown stain) in normal epithelium and adenocarcinomas. Examples of negative, low, moderate and high level staining are shown at $\times 20$ (left) and $\times 40$ (right) magnification. (B) The proportion of patients in each class with negative-low FOXMI staining (hatched bars) and moderate-high FOXMI staining (black bars) in the TMAs is shown. **Represents P-value $=0.04\left(\chi^{2}\right) .(\mathbf{C})$ Box plots of FOXMI protein expression in oesophageal tissue samples from healthy controls, or oesophageal adenocarcinoma patients. Median values of FOXMI are indicated for each tissue type. The box plot demonstrates the interquartile range. ***Represents $P$-value $=0.003$ (T-test). (D) Western blots of FOXMI and PLKI expression in oesophageal adenocarcinoma specimens. The positions of the bands corresponding to FOXMI and PLKI and the locations of the molecular weight markers are shown ( $\mathrm{kDa}$ ). Results of paired normal oesophageal mucosa $(N)$ and tumour $(T)$ specimens or unpaired tumour samples from oesophago-gastrectomy samples are shown. Flol cell lysate is shown in the left lane (bottom panel). ERK2 was used as a loading control.

Figure 3 FOXMI and PLKI mRNA expression in oesophageal adenocarcinomas. (A) RT-PCR analysis of FOXMI (top) and PLKI (bottom) mRNA expression in oesophageal adenocarcinoma samples (right), normal oesophagus from healthy patients (left) and paired samples of normal tissue from patients with oesophageal adenocarcinoma (centre). Data are shown relative to I8S mRNA in oesophageal tissue specimens and are standardised to the expression of the corresponding gene in Hetla cells. The average relative mRNA level and standard deviations derived from two readings from one sample are shown. The individual tissue specimens are numbered and tissue is grouped by tissue subtypes. The mean gene expression for each category of samples is shown in red. (B) Box plots of FOXMI (top) and PLKI (bottom) mRNA expression in oesophageal tissue samples from healthy controls, or oesophageal adenocarcinoma patients. Median values of FOXMI expression are indicated for each tissue type. The box plot demonstrates the interquartile range and the high outliers are indicated. (C) Heatmap showing the relative mRNA expression of total FOXMI or the isoforms FOXM I a, FOXM Ib and FOXMIc (the presence of alternatively spliced exons $A I$ and $A 2$ is shown schematically above) in samples from normal oesophageal tissue and oesophageal adenocarcinoma. mRNA expression is calculated relative to I8S ribosomal RNA. Each horizontal bar represents a single sample. The heatmap was composed based on the mean mRNA expression of the normal oesophageal samples. Low expression is defined as above this mean. Moderate and high expressions are $>$ Is.d. and $>2$ s.d. above this mean. 
levels of FOXM1. Moreover, the high levels of FOXM1 protein seen in $182 \mathrm{~T}, 183 \mathrm{~T}$ and $573 \mathrm{~T}$ samples correlate with the high mRNA levels observed by RT-PCR. Conversely, 557T and 189T samples were negative for both protein and mRNA. We also looked at PLK1 protein levels in a subset of these samples, and found elevated PLK1 levels in two out of four tumour samples (Figure 4D, bottom panel; lanes 5 and 9). Importantly, both of these samples also contained elevated FOXM1 levels, which is consistent with the regulatory inter-relationship between these two proteins.

Taken together, these results demonstrate that FOXM1 is upregulated at both the mRNA and protein levels in a large proportion of oesophageal adenocarcinomas. PLK1 is also commonly upregulated at the mRNA level in oesophageal adenocarcinoma and more limited analysis suggests that this is reflected at the protein level.

\section{The FOXM1 target gene network is upregulated across oesophageal adenocarcinomas}

Our results demonstrate that FOXM1 and its target gene PLK1 are upregulated in a large proportion of oesophageal adenocarcinoma samples. To establish whether there is a correlation in their expression across tumour samples, we compared their relative expression at the mRNA level. High level FOXM1 expression is often correlated with high PLK1 expression (Pearson correlation $=0.730$; Figure $5 \mathrm{~A}$ and $\mathrm{B}$ ). Next, we investigated whether the correlation between FOXM1 and PLK1 expression extended to other FOXM1 target genes; we selected a number of tumour samples with high levels of PLK1 expression and 12 normal samples, and tested for the mRNA expression levels of CCNB1, AURKB and CKS1 (Figure 5B). In general, high FOXM1 levels were associated with a high level expression of all four of its target genes (in 12 out of 14 cases). Little expression of FOXM1 was observed in normal samples, and likewise, minimal or no expression of its target genes was generally observed. To extend this analysis, we took advantage of a recently published study in which the authors used expression microarray analysis to determine the gene expression profiles of 28 normal and 64 oesophageal adenocarcinoma samples from patients who subsequently had their tumours surgically removed (Kim et al, 2010). Preliminary analysis of the expression patterns of FOXM1 and its target genes PLK1, CCNB1, AURKB and CKS1 demonstrated a close correlation of their expression profiles across tumour samples (PCCs all $>0.8$ for pairwise comparisons with FOXM1). We therefore examined the expression profiles of these five genes compared with 100 randomly selected genes. Hierarchal clustering revealed that FOXM1 and its four target genes formed a distinct cluster of genes, thereby confirming their close association of expression pattern among these tumour samples (Figure 6A). Next, we analysed this subgroup of genes in more detail, and this enabled us to segregate the tumour samples into three broad clusters, where all five genes are highly expressed (cluster a), all five genes show low expression (cluster b) and where there is variable expression of these genes (cluster c) (Figure 6B). This spread of expression values is consistent with that demonstrated within our own patient cohort, where some tumour samples show high level coordinate upregulation of FOXM1 and its targets whereas there are other tumours where the expression is generally lower. To establish whether there is any prognostic significance to the expression of FOXM1 and/or its target genes, we monitored survival rates of patients with tumours from clusters a to $c$ (Figure 6C). A Kaplan-Meier plot showed a worsening trend for better survival as the expression of FOXM1 and its target genes increased, that is, cluster A patients fared worse, whereas cluster B patients fared the best. However, the differences failed to reach statistical significance. Interestingly, when the expression of FOXM1 and several of its target genes were analysed individually, high expression levels always produced a trend for shorter survival
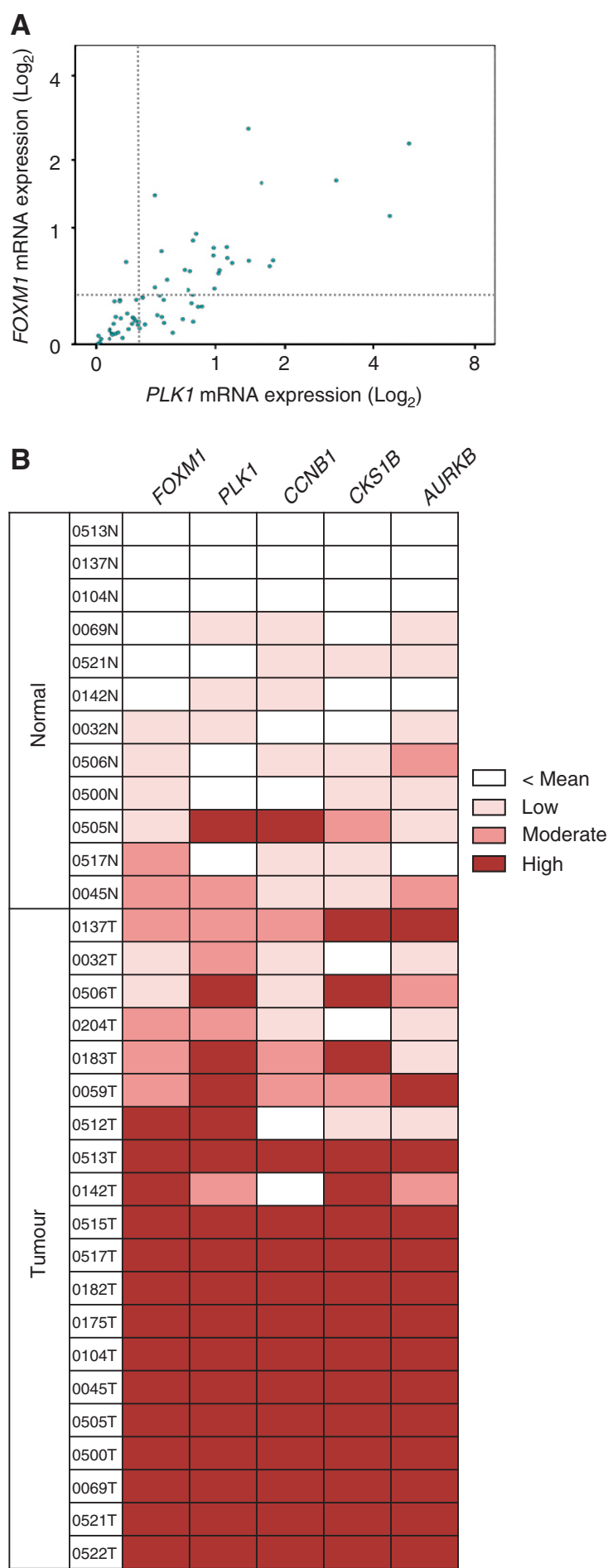

Figure 5 The inter-relationship between FOXMI and its targets in oesophageal adenocarcinomas. (A) Scatter plot of FOXMI vs PLKI mRNA expression levels among samples from oesophageal adenocarcinomas. Data are from Figure $3 A$. Dotted lines represent the gene expression value corresponding to high level expression (i.e., $>2$ s.d. above the mean of the normal samples). (B) Heatmap showing relative mRNA expression of FOXMI, PLKI, CCNBI, AURKB and CKSIB in samples from normal oesophageal tissue and oesophageal adenocarcinoma. mRNA expression is calculated relative to $18 \mathrm{~S}$ ribosomal RNA. Each horizontal bar represents a single sample. The heatmap was composed based on the mean mRNA expression of the normal oesophageal samples. Low expression is defined as above this mean. Moderate and high expressions are $>$ Is.d. and $>2$ s.d. above this mean. 
A
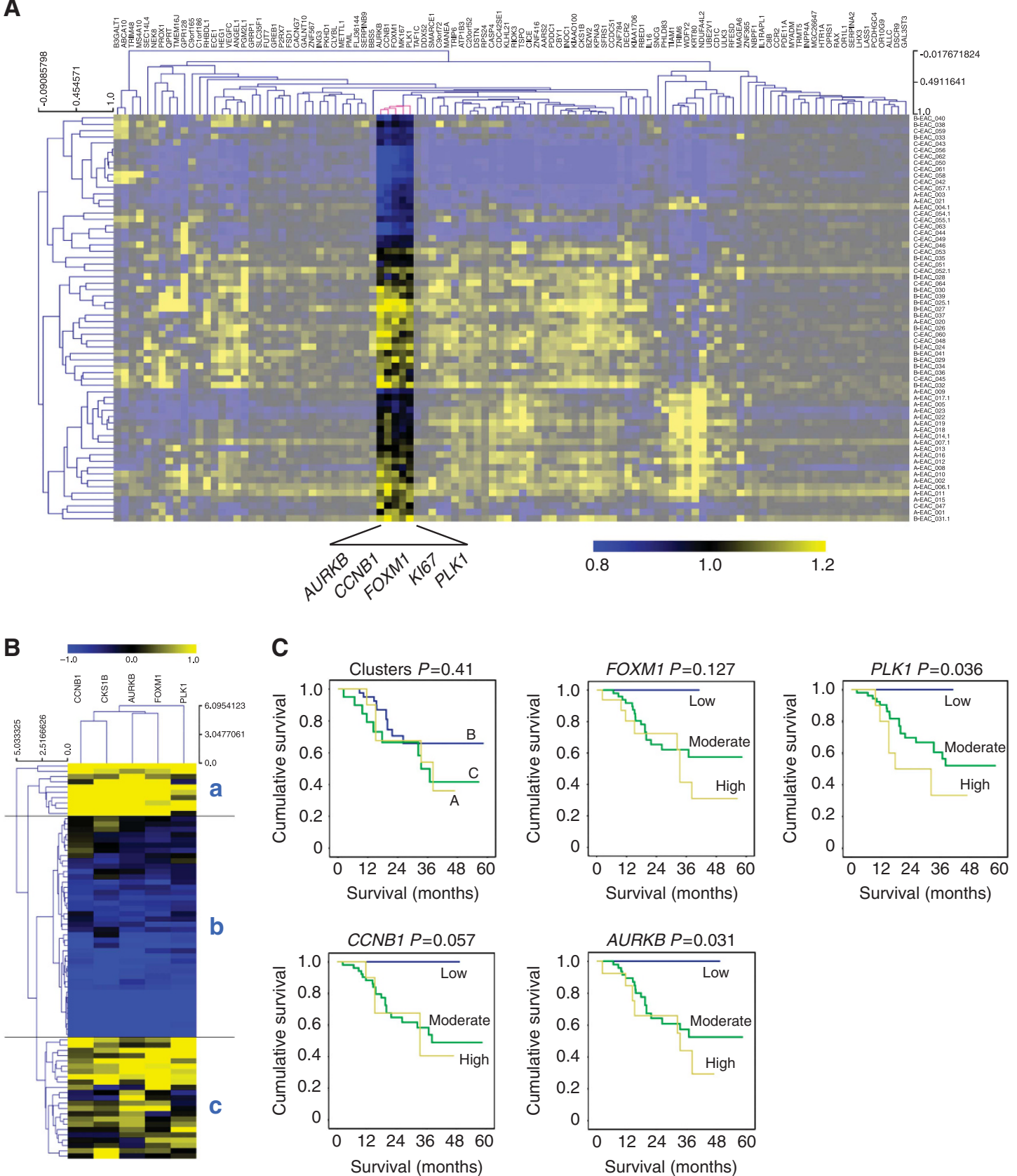

Figure 6 The FOXMI target gene network expression in oesophageal adenocarcinomas. (A) Hierarchal k-means clustering of gene expression levels in oesophageal adenocarcinoma samples. FOXMI, PLKI, CCNBI, AURKB and CKSIB and I00 additional genes taken at random were clustered according to their expression across 78 adenocarcinoma samples (Kim et al, 20l0). A tightly associated subcluster containing FOXMI and several target genes is highlighted (PCCs >0.8). (B) Unsupervised k-means clustering of the expression of FOXMI and the target genes PLKI, CCNBI, CKSI and AURKB in oesophageal adenocarcinoma samples. Three major clusters $(a-c)$ are indicated. (C) Kaplan-Meier curves of overall survival in patients with oesophageal adenocarcinoma, grouped according to the clusters in (B), or due to individual mRNA expression levels for FOXM I, PLKI, CCNB I and AURKB. Expression is defined as low (<Is.d. above mean of normal samples), high ( $>$ Is.d. above mean of normal samples) and moderate (between these values). Log rank probabilities between low and high expression are shown.

times, and in the case of PLK1 and $A U R K B$, the differences in survival rates between patients with high $v s$ low level expression reached statistical significance ( $P$-values 0.036 and 0.031 , respectively).

In summary, these data clearly demonstrate the coordinate upregulation of FOXM1 and its target genes in a subset of oesophageal adenocarcinomas. Furthermore, the analysis of the expression of this cohort of genes has the potential to provide prognostic indicators of survival of patients following surgery.

\section{DISCUSSION}

FOXM1 and PLK1 overexpression has been observed in a variety of different tumour types (reviewed in Strebhardt and Ullrich, 2006 and Koo et al, 2012). However, before this study, their interrelationship within tumours had not been investigated. Here, we have demonstrated that both FOXM1 and PLK1 are commonly overexpressed in oesophageal adenocarcinomas. Thus, FOXM1 and PLK1 overexpression appears to be a frequent event in 
oesophageal tumourigenesis, as previous studies demonstrated that they are overexpressed in oesophageal squamous cell carcinomas (Feng et al, 2009; Zhao et al, 2010; Hui et al, 2012). Importantly, in our study we also found that FOXM1 and PLK1 are generally co-expressed, as might be predicted from their regulatory relationship where FOXM1 controls PLK1 transcription (Laoukili et al, 2005). Indeed, we have also shown that there is coordinate upregulation of other FOXM1 target genes including CCNB1, $A U R K B$ and $C K S 1$, in many oesophageal adenocarcinomas. As both PLK1 and CCNB1 form part of a positive feedback loop that potentiates FOXM1 activity (Major et al, 2004; Fu et al, 2008), this regulatory loop will be potentiated in oesophageal adenocarcinomas. Functionally, all of the FOXM1 target genes that we have analysed are involved in cell-cycle control, chiefly in the late G2 and $M$ phases of the cell cycle, and thus will contribute to the increased proliferative rates seen in the cancer.

Cell line models demonstrated that the FOXM1-PLK1 regulatory axis is operational in oesophageal adenocarcinoma cells. However, little difference was seen in the expression of FOXM1 and PLK1 between adenocarcinoma-derived cell lines, and the non-tumourigenic Hetla cells. However, this was not particularly surprising as it is well established that FOXM1 and its targets are expressed in proliferating cells, as part of the normal physiological control of the cell cycle (reviewed in Laoukili et al, 2007). Subtle differences were however seen in cell-cycle control in adenocarcinoma-derived cell lines, as OE33 and Flo1 cells progressed at different rates through the $\mathrm{G} 2$ and $\mathrm{M}$ phases following release from a double thymidine block. Furthermore, OE33 did not respond well to synchronisation with nocadazole, which probably accounts for the lack of induction of a lower mobility phosphorylated form under these conditions. Overall, the cell line models were not particularly informative about FOXM1 function in cancer cells compared with what is already known about its role in controlling cell-cycle progression.

The FOXM1 transcript undergoes alternative splicing to produce three alternative isoforms (a-c) (Ye et al, 1997). FOXM1b and c are transcriptionally active whereas FOXM1a is transcriptionally inert. All three isoforms are expressed in adenocarcinoma samples but no clear pattern of alternative expression was observed, indicating that no particular isoform is dominant. Thus, transcriptional control of FOXM1 expression rather than alternative splicing is defective in these cancer cells.

Previous studies based on microarray analysis of samples from oesophageal adenocarcinomas have revealed two and four gene signatures that have prognostic value (Kim et al, 2010; Peters et al, 2010). Our studies investigating FOXM1 and its target genes suggest that these genes may also represent useful prognostic indicators. Although analysis of our own samples failed to provide significant correlations with individual clinical parameters, metadata analysis of microarray data derived from patients with adenocarcinomas (Kim et al, 2010) showed that a five gene signature consisting of FOXM1 and four of its target genes was indicative of survival rates in this group of patients. However, although the overall signature failed to reach statistical significance, the analysis of two FOXM1 target genes, PLK1 and AURKB, did indicate that their expression was predictive for long-term survival. Interestingly, a previous study also identified PLK1 mRNA expression as a potential prognostic marker, but this

\section{REFERENCES}

Adams R, Morgan M, Mukherjee S, Brewster A, Maughan T, Morrey D, Havard T, Lewis W, Clark G, Roberts S, Vachtsevanos L, Leong J, Hardwick R, Carey D, Crosby T (2007) A prospective comparison of multidisciplinary treatment of oesophageal cancer with curative intent in a UK cancer network. Eur J Surg Oncol 33: 307-313

Coupland VH, Allum W, Blazeby JM, Mendall MA, Hardwick RH, Linklater KM, Møller H, Davies EA (2012) Incidence and survival of oesophageal finding was not further validated (Peters et al, 2010). These findings raise the possibility that further analysis of FOXM1 and its targets might reveal more robust prognostic indicators of cancer status and progression.

PLK1 inhibitors are currently being developed as cancer therapeutics (reviewed in Lens et al, 2010). As PLK1 has a pivotal role in amplifying the output of the FOXM1 regulatory module, and is itself part of this module that is upregulated in oesophageal adenocarcinomas, this suggests that such inhibitors should be of clinical use in treating patients with oesophageal adenocarcinoma. In particular, the subset of patients that overexpress FOXM1 and its target genes might be particularly susceptible to these inhibitors. Indeed, we have shown that treatment of adenocarcinoma cells with the PLK1 inhibitor BI2536 leads to a reduction in the expression of FOXM1 and its target genes (Figure 2D) and ultimately a block in cell proliferation (data not shown).

\section{CONCLUSIONS}

In summary, this study shows that the FOXM1-PLK1 axis is commonly upregulated in samples from patients with oesophageal adenocarcinoma. Further analysis demonstrates that additional FOXM1 targets are upregulated in these cancer cells. Our results indicate that FOXM1 and its target genes represent molecular markers for this malignancy and candidate novel prognostic indicators of survival.

\section{ACKNOWLEDGEMENTS}

We thank Karren Palmer, Zongling Ji and Michael Jackson for excellent technical assistance; Shen-Hsi Yang and members of our laboratory for comments on the manuscript and stimulating discussions. This work was supported by grants from Research and Development (ABJ0026) and the Cancer Therapy and Research Fund from the WWL NHS Foundation Trust and from the department of Gastroenterology to RK and YSA, the UK NIHR/ UKCRN (UK National Institute of Health Research/Cancer Research Network) to YSA, Manchester Experimental Cancer Medicine Centre Funding and grants from the Wellcome Trust and a Royal Society-Wolfson award to ADS.

\section{Conflict of interest}

The authors declare no conflict of interest.

\section{Author contributions}

MD contributed to the study design, conducted the majority of the experiments and helped with manuscript preparation. JC and $\mathrm{SH}$ assisted with tissue sampling and IHC analysis. HV and CW assisted with the TMA experiments. NH performed the bioinformatics analysis. AY provided clinical training, contributed to study design and coordination and data interpretation. ADS contributed to experimental design and coordination and wrote the manuscript. All authors read and approved the final manuscript. and gastric cancer in England between 1998 and 2007, a populationbased study. BMC Cancer 12: 11

Feng Y-B, Lin D-C, Shi Z-Z, Wang X-C, Shen X-M, Zhang Y, Du X-L, Luo M-L, Xu X, Han Y-L, Cai Y, Zhang Z-Q, Zhan Q-M, Wang M-R (2009) Overexpression of PLK1 is associated with poor survival by inhibiting apoptosis via enhancement of survivin level in esophageal squamous cell carcinoma. Int J Cancer 124: 578-588 
Fu Z, Malureanu L, Huang J, Wang W, Li H, van Deursen JM, Tindal DJ, Chen J (2008) Plk1-dependent phosphorylation of FoxM1 regulates a transcriptional programme required for mitotic progression. Nat Cell Biol 10: 1076-1082

Hampel H, Abraham NS, El-Serag HB (2005) Meta-analysis: obesity and the risk for gastroesophageal reflux disease and its complications. Ann Intern Med 143: 199-211

Hui MKC, Chan KW, Luk JM, Lee NP, Chung Y, Cheung LCM, Srivastava G, Tsao SW, Tang JC, Law S (2012) Cytoplasmic forkhead Box M1 (FoxM1) in esophageal squamous cell carcinoma significantly correlates with pathological disease stage. World J Surg 36: 90-97

Keld R, Guo B, Downey P, Gulmann C, Ang YS, Sharrocks AD (2010) The ERK MAP kinase-PEA3/ETV4-MMP-1 axis is operative in oesophageal adenocarcinoma. Mol Cancer 9: 313

Kim SM, Park Y-Y, Park ES, Cho JY, Izzo JG, Zhang D, Kim S-B, Lee JH, Bhutani MS, Swisher SG, Wu X, Coombes KR, Maru D, Wang KK, Buttar NS, Ajani JA, Lee J-S (2010) Prognostic biomarkers for esophageal adenocarcinoma identified by analysis of tumor transcriptome. PLoS ONE 5: e15074

Koike T, Ohara S, Sekine H, Iijima K, Abe Y, Kato K, Toyota T, Shimosegawa T (2001) Helicobacter Pylori infection prevents erosive reflux oesophagitis by decreasing gastric acid secretion. Gut 49: 330-334

Koo C-Y, Muir KW, Lam EW (2012) FOXM1: from cancer initiation to progression and treatment. Biochim Biophys Acta 1819: 28-37

Lao-Sirieix P, Boussioutas A, Kadri SR, O’Donovan M, Debiram I, Das M, Harihar L, Fitzgerald RC (2009) Non-endoscopic screening biomarkers for Barrett's oesophagus: from microarray analysis to the clinic. Gut 58: 1451-1459

Laoukili J, Kooistra MRH, Brás A, Kauw J, Kerkhoven RM, Morrison A, Clevers H, Medema RH (2005) FoxM1 is required for execution of the mitotic programme and chromosome stability. Nat Cell Biol 7: 126-136

Laoukili J, Stahl M, Medema RH (2007) FoxM1: at the crossroads of ageing and cancer. Biochim Biophys Acta 1775: 92-102

Lens SMA, Voest EE, Medema RH (2010) Shared and separate functions of polo-like kinases and aurora kinases in cancer. Nat Rev Cancer 10: 825-841

Major ML, Lepe R, Costa RH (2004) Forkhead Box M1B transcriptional activity requires binding of Cdk-Cyclin complexes for phosphorylation- dependent recruitment of p300/CBP coactivators. Mol Cell Biol 24: 2649-2661

Peters CJ, Rees JRE, Hardwick RH, Hardwick JS, Vowler SL, Ong C-AJ, Zhang C, Save V, O'Donovan M, Rassl D, Alderson D, Caldas C, Fitzgerald RC (2010) A 4-gene signature predicts survival of patients with resected adenocarcinoma of the esophagus, junction, and gastric cardia. Gastroenterology 139: 1995-2004

Raychaudhuri P, Park HJ (2011) FoxM1: a master regulator of tumor metastasis. Cancer Res 71: 4329-4333

Saeed AI, Bhagabati NK, Braisted JC, Liang W, Sharov V, Howe EA, Li J, Thiagarajan M, White JA, Quackenbush J (2006) TM4 microarray software suite. Methods Enzymol 411: 134-193

Solaymani-Dodaran M, Logan RFA, West J, Card T, Coupland C (2004) Risk of oesophageal cancer in Barrett's oesophagus and gastrooesophageal reflux. Gut 53: 1070-1074

Strebhardt K, Ullrich A (2006) Targeting polo-like kinase 1 for cancer therapy. Nat Rev Cancer 6: 321-330

Whitfield ML, Zheng L-X, Baldwin A, Ohta T, Hurt MM, Marzluff WF (2000) Stem-loop binding protein, the protein that binds the $3^{\prime}$ end of histone mRNA, is cell cycle regulated by both translational and posttranslational mechanisms. Mol Cell Biol 20: 4188-4198

Ye H, Kelly TF, Samadani U, Lim L, Rubio S, Overdier DG, Roebuck KA, Costa RH (1997) Hepatocyte nuclear factor 3/fork head homolog 11 is expressed in proliferating epithelial and mesenchymal cells of embryonic and adult tissues. Mol Cell Biol 17: 1626-1641

Zhang N, Wei P, Gong A, Chiu WT, Lee HT, Colman H, Huang H, Xue J, Liu M, Wang Y, Sawaya R, Xie K, Yung WK, Medema RH, He X, Huang S (2011) FoxM1 promotes $\beta$-catenin nuclear localization and controls Wnt target-gene expression and glioma tumorigenesis. Cancer Cell 20: 427-442

Zhao C, Gong L, Li W, Chen L (2010) Overexpression of Plk1 promotes malignant progress in human esophageal squamous cell carcinoma. $J$ Cancer Res Clin Oncol 136: 9-16

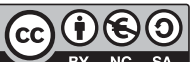

This work is licensed under the Creative Commons Attribution-NonCommercial-Share Alike 3.0 Unported License. To view a copy of this license, visit http:// creativecommons.org/licenses/by-nc-sa/3.0/ 